\title{
A Novel Piezoelectric Micro-gripper's Control Based on Modified Smith Predictor
}

\author{
Xiangjin ZENG*, Jimin WANG, Pengpeng SONG, Sisi HUANG \\ ${ }^{1}$ Hubei Province Key Laboratory of Intelligent Robot \\ ${ }^{2}$ School of Computer Science and Engineering \\ Wuhan Institute of Technology \\ 430205 Wuhan, China \\ E-mail: xjzeng21@163.com \\ +* Corresponding author
}

\begin{abstract}
We develop a micro-gripper that actuated by piezoelectric cantilever for the need of micro parts assembly. The displacement-voltage relationship model is given in this paper. For hysteresis of piezoelectric ceramic, a control scheme based on fuzzy adaptive PID with a Modified Smith Predicator for the control of micro-gripper is proposed. The results of micro-assembly prove that the model is efficiency and feasibility.
\end{abstract}

Keywords-piezoelectric cantilever modeling; micro gripper; modified Smith predictor; fuzzy adaptive PID

\section{INTRODUCTION}

We must design the end-effecter for micro-surgery, IC assembly. Since the size of the object is generally micrometer or nanometer level, the design of the end-effecter has become an important research topic in the field of micro manipulation, with the requirement with a flexible attitude adjustment, positioning, capture, release and other operations. As the requirements of high-precision positioning for operational objects, the displacement control of end-effecter becomes an important prerequisite for automatic micro-assembly[1].

However, the Piezoelectric micro-gripper exists hysteresis and the control is non-linear. Japan Tanikawa , who proposed $\mathrm{H}_{\infty}$ controller to achieve the robustness control and the hysteresis compensation for two-finger micro hand, but it is difficult to obtain a fast system response. Paper [2] [3] [4]apply the typical Preisach model to compensate the impact of the piezoelectric hysteresis, but it has a litter effect; In the paper[5], it established a flexible hinge of the piezoelectric hysteresis model, the control model is simple and control is better, but closed-loop control is not achieved.

For the above problem, In this paper, we build the relationship model of displacement and voltage of piezoelectric-driven micro-gripper, and we propose the hybrid PID control algorithm based on Modified Smith Predicator feedforward compensation control for hysteresis[6]-[9]. The Micro-assembly has been proved effective control of the model structure, and have good reliability and validity..

\section{The Structure OF MiCRO-GRIPPER}

We employ the piezoelectric micro-gripper that uses the curved type piezoelectric bimorph cantilever as finger, two fingers with symmetrical structure. In order to achieve micro-power testing, the piezoelectric bimorph paste the strain gauge. From the analysis of materials mechanics, the cantilever root has the greatest moment when press an external force for the end of the cantilever. So we paste the strain gauges in the root of the bimorph cantilever to obtain the maximize strain signal. The micro-gripper is shown in Fig 1 .

As a result of the two-finger symmetry structure, we can employ a single piezoelectric bimorph cantilever beam characteristics to study.

\section{The RELATIONSHIP MODELING BETWEeN \\ DiSPLACEMENT AND VOLTAGE OF MICRO-GRIPPER}

The Piezoelectric bimorph cantilever beam is used two parallel piezoelectric ceramic to paste with the opposite polarization. The structure is shown in Fig.2. we ignores the thickness of the middle layer.

As the beam length is much greater than its width and the second stress tensor of cantilever is $\mathrm{T} 2=\mathrm{T} 3=\mathrm{T} 4=\mathrm{T} 5=\mathrm{T} 6=$ 0 ; the strain tensor is $\mathrm{S} 1, \mathrm{~S} 2, \mathrm{~S} 3, \mathrm{~S} 4, \mathrm{~S} 5, \mathrm{~S} 6 \neq 0$. So according to the first piezoelectric equation[7], we can obtain the strain of piezoelectric layer S1:

$$
S_{1}=S_{11}^{E} T_{1}+s_{12}^{E} T_{2}+s_{13}^{E} T_{3}+\ldots+d_{31} E_{3}=s_{11}^{E} T_{1}+d_{31} E_{3}
$$

Where $S_{i j}^{E} \quad(\mathrm{i}=1,2,3, \mathrm{j}=1,2,3)$ is the elastic compliance coefficient in the constant electric field E; and $d_{31}$ is the piezoelectric strain coefficient of piezoelectric body; and $E_{3}$ is the electric field. Then the piezoelectric layer T1 stress tensor is:

$$
T_{1}=\left(s_{11}^{E}\right)^{-1} S_{1} \pm\left(s_{11}^{E}\right)^{-1} d_{31} E_{3}=E_{p} S_{1} \pm E_{p} d_{31} E_{3}
$$

Where $E_{\mathrm{p}}$ is the elastic modulus. In Fig.2, we set the origin coordinates of center cross-section of piezoelectric ceramics, $y$ is the distance from the center, $l$ is the width of beam cross-section, $h$ is the thickness of each piezoelectric ceramic.

According to the moment balance equation of the material mechanics 


$$
M=\int_{h_{u p}} T_{1} l d y+\int_{h_{\text {down }}} T_{1} l d y=0
$$

then

$$
\int_{-h}^{0}\left(E_{\mathrm{p}} y \rho+E_{\mathrm{p}} d_{31} E_{3}\right) l y d y+\int_{0}^{h}\left(E_{\mathrm{p}} y \rho-E_{\mathrm{p}} d_{31} E_{3}\right) l y d y=0(4)
$$

where $\rho$ is the type plane curvature when the cantilever is pure bending deformation.

We use the $E_{3}=\mathrm{U} / h$ into (4):

$$
\rho=\frac{3 E_{\mathrm{p}} d_{31} U}{2 E_{\mathrm{p}} h^{2}}
$$

where $\mathrm{U}$ is the piezoelectric ceramic voltage.

Can see from the disturbing degree equation: $\ddot{y}(x)=\rho$, the relationship model between $y$ and voltage $\mathrm{U}$ is:

$$
y=\frac{3 E_{\mathrm{p}} d_{31} U}{4 E_{\mathrm{p}} h^{2}} l_{\mathrm{pze}}{ }^{2}
$$

Where $l_{\text {pze }}$ is the length of the cantilever free-end.

\section{HYSTERESIS MODELING AND MODIFIED SMITH PREDICTOR}

As the complexity of piezoelectric ceramic structure and working mechanism, the external electric field strength and the piezoelectric ceramic displacement, strain have been the creep, hysteresis and non-linear and other undesirable characteristics, as shown in Fig. 3.

The crystal polarization effect causes generally hysteresis. However, the hysteresis is a negative factor for high precision positioning control. Therefore, we design Fuzzy PID closed loop control algorithm based on the smith predictor.

\section{A. The Principle Diagram of Smith Predictor}

Fig.4 shows the principle of. The control system consists of controller $G_{c}(S)$, predictor $G_{m}(S)\left(1-e^{-r s}\right)$, control object $G_{o}(S) e^{-r s}$.

Under condition of predictor model matching object model, namely, $G_{o}(S)=G_{m}(S)$, The transfer function of control system can be represented as (7).

$$
\Phi(s)=\frac{G_{c}(S) G_{o}(s) e^{-\tau s}}{1+G_{c}(S) G_{o}(s)}
$$

It can be seen from (7) that delay link $e^{-r s}$ is not located in close loop control link, meaning that hysteresis delay can not affect the stability of control system. Delay link $e^{-r s}$ defers only time $r$ for control system and the control performance is same as the object model with transfer function $G_{o}(S)$. Therefore, Smith predictor eliminates the hysteresis delay and improves the performance.

\section{B. Fuzzy Adaptive PID Control with a Modified Smith Predictor}

Generally, the structure of position based micro-gripper can be constructed as Fig.5. Firstly, Control system obtains object position r(s) and end-effector position c(s) using image processing. Secondly, employs the trajectory planning as feedback to predict the next movement for manipulator. In real task, although robotic current position can be obtained from joint feedback, we need that estimate relative position of object and end-effector for calibration error, position estimation error and system error. So, we present a control scheme based on fuzzy adaptive PID with a modified smith predicator to offset hysteresis[10]-[12].

The structure with modified smith predictor is shown in Fig.6. We give that the transfer function of micro-gripper is $\mathrm{Gr}$ and the transfer function of PID controller is G with $\mathrm{Kp}, \mathrm{Ki}, \mathrm{Kd}$ from fuzzy deduction. $G_{m}(S)\left(1-e^{-r s}\right)$ is the transfer function of smith predictor. D is the transfer function of disturbance. $\mathrm{M}$ is a new added the transfer function of disturbance rejection. It can be seen from Fig.4 that control system is a standard smith predictor when $\mathrm{M}$ is lacked in system structure.

The response of control system can be written as (8) when meets $G_{m}(S)=G_{r}(S)$ :

$$
C(S)=T_{1}(S) R(S)+T_{2}(S) D(S)
$$

where

$$
\begin{gathered}
T_{1}(S)=\frac{G_{m} G e^{-r s}}{1+G_{m} G} \\
T_{2}(S)=\frac{\left[1+G_{m} G-G_{m} G e^{-r s}\right] G_{m} e^{-r s}}{\left(1+G_{m} G\right)\left(1+M G_{m} e^{-r s}\right)}
\end{gathered}
$$

According to (9), Characteristic equation has not delay link, then PID controller can be decided only by system itself. In addition, the response of disturbance can be also controlled only by the new-added controller M. It has been shown[10] that the smith predictor gives a steady state error under disturbances for open loop unstable process. Clearly, the new-added controller $\mathrm{M}$ can improve the system performance of disturbance rejection. Next, the control process based new structure will be given.

In the micro-operations, the piezoelectric bimorph cantilever can be modeled by mass - spring - damper system. The system can be characterized as follows: 


$$
m \ddot{x}+c \dot{x}+k x+F_{h y}=F
$$

Where $m$ is the mass of the bimorph cantilever beam, $c$ is damper, the $k$ is the stiffness; $F, F_{h y}$ is the self-generated force of piezoelectric bimorph and the produced force during the process of hysteresis respectively; $X$ is the displacement of cantilever deflection.

Therefore, we can use the improved smith predictor to build the produced force during the process of hysteresis:

$$
\begin{aligned}
& \frac{d q}{d t}=A q \frac{d x}{d t}+B u \frac{d x}{d t} \\
& F_{h y}=C q \\
& \mathrm{~A}=\left[\begin{array}{cc}
0 & 1 \\
-\left(\frac{2 \pi}{T}\right)^{2}-\left(\frac{2}{T} \ln \left(\frac{P_{n-1}}{P_{n}}\right)\right)^{2} & -\operatorname{sgn}(d x) d t) \frac{2}{T} \ln \left(\frac{P_{n-1}}{P_{n}}\right)
\end{array}\right] \\
& \mathrm{B}=\left[\begin{array}{c}
\mathrm{O} \\
1
\end{array}\right] \\
& C=\left[G\left(\left(\frac{2 \pi}{T}\right)^{2}+\left(\frac{2}{T} \ln \left(\frac{P_{n-1}}{P_{n}}\right)\right)^{2}\right) \operatorname{sgn}(d x) S\right]
\end{aligned}
$$

Where $u$ is the input voltage, $\mathrm{T}$ is the second-order damped response oscillations period, $P_{n}$ is the amplitude of deviation from second order oscillation steady-state response , $\mathrm{G}$ is the DC voltage gain, $\mathrm{S}$ is the initial slope.

Then (11) can be written as second-order damped oscillation system:

$$
\left\{\begin{array}{c}
\dot{x}=R x+S u+W F_{h y} \\
y=Q x
\end{array}\right.
$$

\section{Parameter Identification and PID Closed Loop Control}

Assume that the system is less damping system,

$$
\begin{aligned}
\xi=0.04 A & =\left[\begin{array}{cc}
0 & 1 \\
-0.02 & -0.052
\end{array}\right] \quad B=\left[\begin{array}{l}
0 \\
1
\end{array}\right] \\
C & =\left[\begin{array}{ll}
4.02 & 0.011
\end{array}\right]
\end{aligned}
$$
(17).

Therefore, we can determine the system parameters of

We design a two DOF controller based fuzzy adaptive PID control law for micro-gripper.

The fuzzy adaptive PID controller applies error e and error change ec as its input. Then, we build a fuzzy rule table based practice experiences, which gives the counterpart relationship between $\mathrm{Kp}, \mathrm{Ki}, \mathrm{Kd}$ and error e , error change ec. So, we can revise online control system parameters. (18) shows the revised computation formula.

$$
\begin{aligned}
& K_{p}=K_{p}^{*}+\left\{e_{i}, e c_{i}\right\}_{p} \\
& K_{i}=K_{i}^{*}+\left\{e_{i}, e c_{i}\right\}_{i} \\
& K_{d}=K_{d}^{*}+\left\{e_{i}, e c_{i}\right\}_{d}
\end{aligned}
$$

Then, the control output can be represented as (19).

$$
u(t)=\hat{J}_{v}^{-1}(t) \cdot u^{\prime}(t)=\hat{J}_{v}^{-1}(t) \cdot\left[K_{P} \tilde{\xi}(t)+\beta K_{l} \sum_{j=0}^{t} \tilde{\xi}(j) T+K_{D} \frac{\tilde{\xi}(t)-\tilde{\xi}(t-1)}{T}\right]
$$

Where $u(t)$ is the control output of controller in task space and $\hat{J}_{v}(t)$ is jacobian matrix.

\section{EXPERIMENT}

Now, we use the piezoelectric bimorph cantilever as the experimental object. Young's modulus is $E=7 \times 10^{10} \mathrm{~N} / \mathrm{m}$, the beam size is $40 \times 2 \times 0.2 \mathrm{~mm}^{3}$

We can obtain the relationship of micro-gripper displacement and voltage according to the ideal model: $y=2 \mathrm{~V}$

Piezoelectric bimorph drive power increments from $0 \mathrm{~V}$ to $75 \mathrm{~V}$, while measures the displacement of the free end of piezoelectric bimorph. When reach the maximum voltage and then decrements gradually voltage. After repeated measurements, we can draw the curve as shown in Fig.7. It can be seen from Fig.7 that in theory-driven displacement and driving voltage is linear, but it is a big delay when the voltage increments process and the voltage decrements process.

Joining the feedforward Fuzzy PID closed loop controller based the modified smith predictor; the system linearity is improved significantly, and compensates the hysteresis characteristics of the piezoelectric actuator. Then, we test the tracking experiments based the proposed method. Fig. 8 shows the tracking results of fuzzy PID controller. Fig.9 shows the tracking error using feedforward fuzzy PID closed loop controller based modified smith predictor. We can seen from the Fig.8 that the feedforward fuzzy PID closed loop controller based modified smith predictor is better, inhibited significantly the hysteresis effect of piezoelectric cantilever beam.

\section{CONCLUSION}

We developed a micro-gripper based on piezoelectric, and established the relationship model of displacement and voltage of micro-gripper. For piezoelectric ceramic hysteresis, we proposed the hybrid PID control algorithm based on modified smith predictor feedforward compensation control. Then, we test the tracking experiments. The results show that modified smith predictor feedforward and fuzzy PID closed-loop tracking controller is better, inhibited significantly 
the impact of the hysteresis effect of the piezoelectric cantilever and achieve control requirements.

\section{ACKNOWLEDGMENT}

Supported by the National Natural Science Foundation of China (11101318), the key project fund of Hubei Provincial Department of education, Hubei Provincial Key Laboratory of intelligent robots (HBIR201705), the Science Foundation of WIT(K201476).

\section{REFERENCES}

[1] Hui Xie, Lining Sun, Weibin Rong and Xiufeng Yuan. "Visual servoing with modified smith predictor for micromanipulation tasks," Proceedings of the IEEE international conference on mechatronics \& automation, pp.71-76, July,2005

[2]Wei. T. A., Garmon F.A., Khosla P.K., et al. Modeling rate-dependent hysteresis in piezoelectric actuators [J]. Proceedings of IEEE/RSJ International Conference on Intelligent Robots and Systems, 2003, 2:1975-1980.

[3] Gorbert R. B., Morris K.A., Wang D.W.L. Passivity-based Stability and Control of Hysteresis in Smart Actuators [J]. IEEE Transactions on Control Systems Technology, 2001, 9(1):5-16.

[4] Cahyadi A.I., Yamamoto Y. Modeling a Micro Manipulation System with Flexure Hinge [J]. IEEE Conference on Robotics, Automation and Mechatronics,2006,2: 1-5

[5] Sun R.X., Chang T. Control of Hysteresis in a Monolitic Nanoactuator [J]. Proceeding of American Control Conference, 2001,3:2261-2266.

[6] Yi De Chen, Pi Cheng Tung, Chyun Chau Fuh. "Modified smith predictor scheme for periodic disturbance reduction in linear delay systems,” Journal of Process Control, Vol. 17, pp.799-804,2007.

[7] Sun Kang, Zhang University. Piezoelectricity [M]. Beijing: National Defence Industry Press,1984.

[8] Smits J.G., Choi,W. The Constituent Equations of Piezoelectric Heterogeneous Bimorphs[J]. IEEE transactions on ultrasonic ferroelectrics and frequency control, 1991, 38(3):256-270.

[9] Sun R.X., Chang T. Control of Hysteresis in a Monolitic Nanoactuator [J]. Proceeding of American Control Conference, 2001,3:2261-2266.

[10] Ibrahim Kaya. "IMC based automatic tuning method for PID controllers in a Smith predictor configuration," Computers and Chemical Engineering, Vol 28, pp.281-290, 2004.

[11] Liu Jinkun. “Advance PID control and MATLAB simulation,”.Beijing: Electronics Industry Publishing House,2003

[12] FERREIRA A , CASSIER C , HIRA S. "Automatic microassembly system assisted by vision servoing and virtual reality," IEEE T ransactions on Mechatronics, Vol. 9, No.2, pp. 321-323,2004.

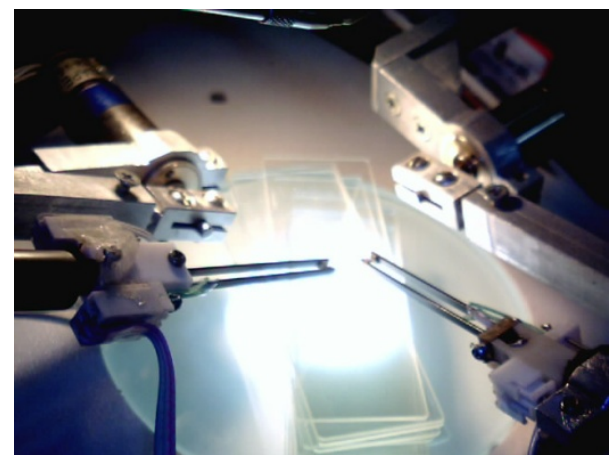

Figure 1. The piezoelectric micro-gripper

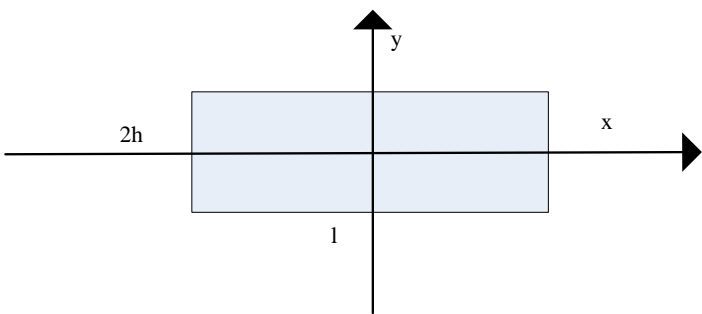

Figure 2. The beam cross-section

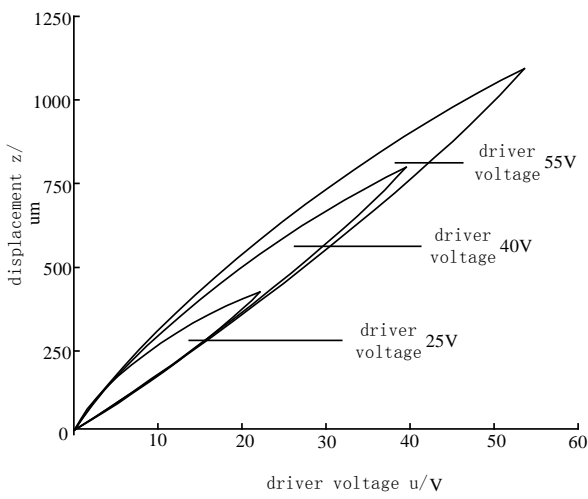

Figure 3. The hysteresis loop under different peak voltage

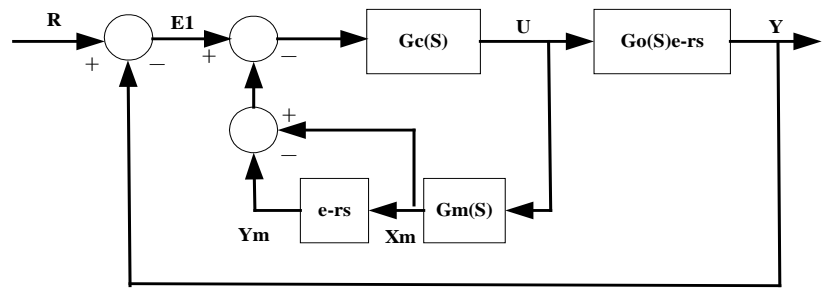

Figure 4. The principle diagram of smith predictor

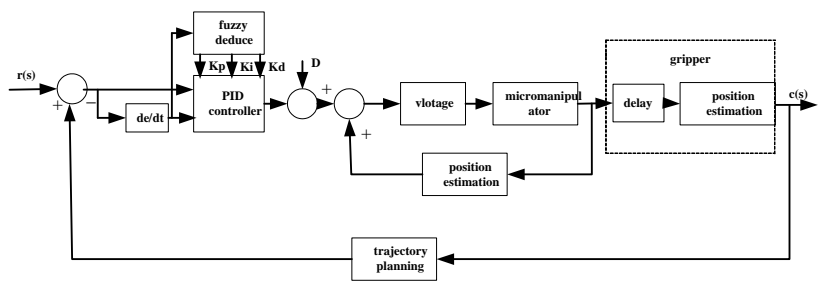

Figure 5. The structure of position

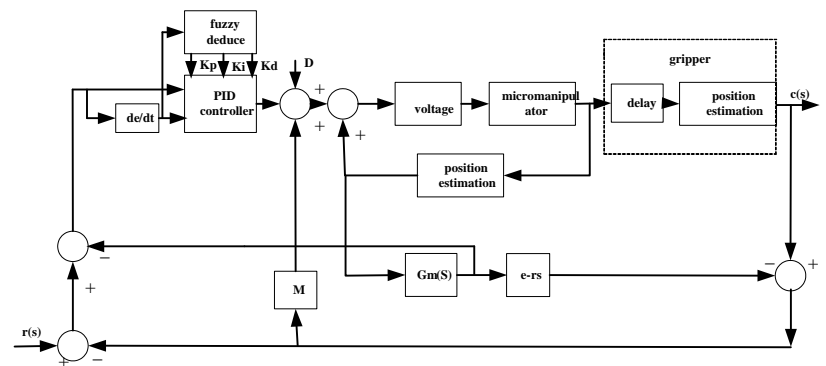

Figure 6 . The structure with modified smith predictor 


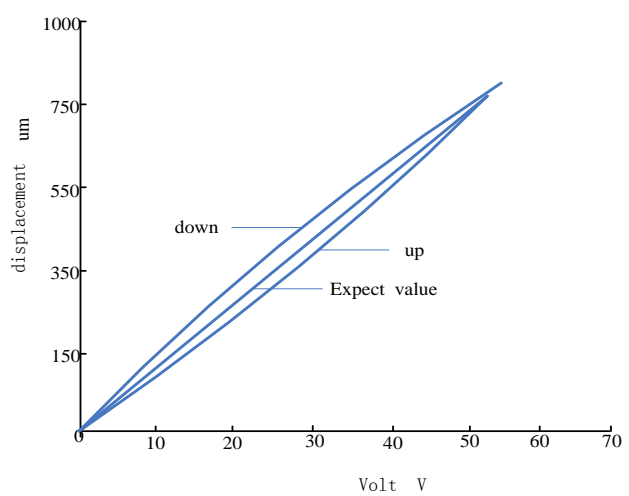

Figure 7. The voltage - displacement curve

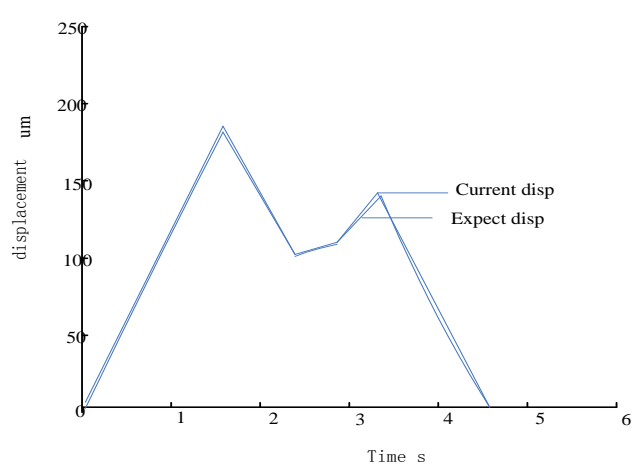

Figure 8. The Fuzzy PID controller, the tracking results

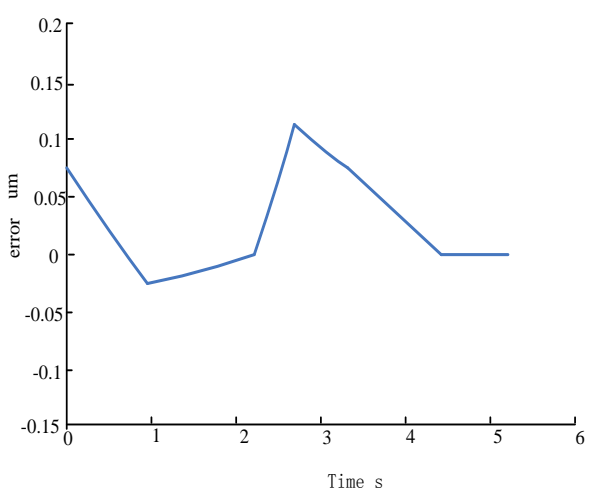

Figure 9. The modified smith predictor of fuzzy PID tracking error 